\title{
Ghost Free Analysis of a Nonsymmetric Theory of Gravitation
}

\author{
S. Ragusa and L. Chibebe Céleri \\ Departamento de Física e Informática, Instituto de Física de São Carlos \\ Universidade de São Paulo, C.P. 369, 13560-970, São Carlos, SP, Brazil
}

Received on 5 June, 2003

\begin{abstract}
The absence of ghost-negative energy radiative modes in a proposed generalized theory of gravitation based on a non-symmetric metric is reanalyzed. The missing contribution of the symmetric sector of the Lagrangian is calculated and shown to be null. The ghost free character of the theory is then firmly established.
\end{abstract}

\section{Introduction}

In this paper we shall investigate in a more complete way the problem of the emission of gravitational radiation in a metric nonsymmetric theory of gravitation developed by one of us in a previous work [1], herefrom referred to as I, which has not been adequately investigated in that paper. In a second paper [2] the solution of the field equations for a spherical point source was obtained together with its implications for the motion of test particles and light. The theory was shown to be consistent with the four classical solar tests of general relativity.

The sources of the field are the energy-momentumstress tensor $T_{\alpha \beta}$ and the phenomenological coupled matter fermion number current density $S^{\alpha}=F n^{\alpha}$, where $n^{\alpha}=n u^{\alpha}$ is the fermion number density $(n)$ current found in the description of the interior of stars and $F$ is the coupling constant of the current to the geometry. The gravitational radiation comes from both of these sources, $S^{\alpha}$ being the one connected directly to the antisymmetric part $g_{[\alpha \beta]}$ of the metric. In I it was claimed that the energy of the emitted radiation coming from the current source $S^{\alpha}$ is positive definite. As the part coming from the stress $T_{\alpha \beta}$ has no problem, it was concluded that the theory is free of ghostnegative radiative modes which, as shown by Damour, Deser and McCarthy (DDM) [3], were present in previous theories. However, in analyzing the radiation coming from the current source $S^{\alpha}$ only a part of the Lagrangian, its antisymmetric sector, was taken into account leaving it open the question of what the contribution coming from the other part, the symmetric sector, might be. Here we complete this missing calculation showing that this last contribution is actually null guaranteeing in this way that the theory is really ghost free.

\section{The field equations}

The vacuum field Lagrangian of the theory is written in Eq. (I-5.13), here with the replacement $2 \Delta_{\alpha} / 3=\Gamma_{\alpha}$,

$$
\mathbf{L}=\mathbf{g}^{\alpha \beta}\left(U_{\alpha \beta}+\Gamma_{[\alpha, \beta]}+\frac{1}{2} \Lambda g_{\alpha \beta}\right) .
$$

Here we use the notation $\mathbf{X}=\sqrt{-g} X$, where $g$ is the determinant of $g_{\alpha \beta}$ whose inverse is $g^{\alpha \beta}$ as defined by $g^{\alpha \beta} g_{\alpha \gamma}=$ $\delta_{\gamma}^{\alpha} . \mathrm{Next}$

$$
U_{\alpha \beta}=\Gamma_{(\alpha \beta), \sigma}^{\sigma}-\Gamma_{(\sigma \alpha), \beta}^{\sigma}+\Gamma_{(\alpha \beta)}^{\sigma} \Gamma_{(\sigma \lambda)}^{\lambda}-\Gamma_{(\alpha \lambda)}^{\sigma} \Gamma_{(\sigma \beta)}^{\lambda},
$$

symmetric, because the second term is (see below), is the analogue of the Ricci tensor, depending only on the symmetric part of the connection which is given by

$$
\begin{aligned}
& \Gamma_{(\alpha \beta)}^{\sigma}=\frac{1}{2} g^{(\sigma \lambda)}\left(s_{\alpha \lambda, \beta}+s_{\lambda \beta, \alpha}-s_{\alpha \beta, \lambda}\right) \\
& +\frac{1}{4}\left(g^{(\sigma \lambda)} s_{\alpha \beta}-\delta_{\alpha}^{\sigma} \delta_{\beta}^{\lambda}-\delta_{\alpha}^{\lambda} \delta_{\beta}^{\sigma}\right)\left(\ln \frac{s}{g}\right)_{, \lambda},
\end{aligned}
$$

where $s_{\alpha \beta}$ symmetric and with determinant $s$, is the inverse of $g^{(\alpha \beta)}$ as defined by $s_{\alpha \beta} g^{(\alpha \gamma)}=\delta_{\beta} \gamma$. Next, $\Gamma_{\alpha}=$ $\frac{1}{2}\left(\Gamma_{\alpha \mu}^{\mu}-\Gamma_{\mu \alpha}^{\mu}\right)$ is the torsion vector involving contractions of the antisymmetric part of the connection and $\Lambda$ is the cosmological constant. The $\Lambda$ term of Eq. (1) could be written $2 \sqrt{-g} \Lambda$ but we shall keep it as it is to make a close contact to I. From Eq. (3) one sees that $\Gamma_{(\sigma \alpha)}^{\sigma}=(\ln (-g) / \sqrt{-s})_{, \alpha}$ so the second term on the right of Eq. (2) is in fact symmetric. Variations with respect to $\mathrm{g}^{\alpha \beta}, \Gamma_{(\alpha \beta)}^{\gamma}$ and $\Gamma_{\alpha}$ yields the field equations. The former leads to $U_{\alpha \beta}+\Gamma_{[\alpha, \beta]}+\Lambda g_{\alpha \beta}=0$, with symmetric and antisymmetric parts given by

$$
U_{\alpha \beta}+\Lambda g_{(\alpha \beta)}=0
$$

and

$$
\Gamma_{[\alpha, \beta]}+\Lambda g_{[\alpha \beta]}=0 .
$$

The variation with respect to $\Gamma_{(\alpha \beta)}^{\sigma}$ leads to the third field equation

$$
\mathbf{g}^{(\alpha \beta)}{ }_{, \gamma}+\mathbf{g}^{(\alpha \mu)} \Gamma_{(\mu \gamma)}^{\beta}+\mathbf{g}^{(\beta \mu)} \Gamma_{(\mu \gamma)}^{\alpha}-\mathbf{g}^{(\alpha \beta)} \Gamma_{(\mu \gamma)}^{\mu}=0,
$$

which can be solved as in Eq. (3), and the last one leads to the fourth one,

$$
\mathbf{g}^{[\alpha \beta]}, \beta=0 .
$$




\section{The energy-momentum-stress ten- sor}

Following DDM [4], the idea behind the calculation is to expand the field Lagrangian in Eq.(1) about a Riemannian general relativity (GR) background space with metric $G_{\alpha \beta}$ in such a way as to have the Lagrangian splitted in two terms, the one of GR and a second one containing the contribution of $g_{[\alpha \beta]}$. This is done by writing $g_{\alpha \beta}=G_{\alpha \beta}+\bar{g}_{\alpha \beta}$ and expand $\bar{g}_{\alpha \beta}=\bar{g}_{(\alpha \beta)}+\bar{g}_{[\alpha \beta]}$ in powers of the lowest-order antisymmetric part of the metric $B_{\alpha \beta}=\bar{g}_{[\alpha \beta]}^{(1)}$ which acts as a perturbation, keeping the expansion to second order

$$
g_{\alpha \beta}=G_{\alpha \beta}+B_{\alpha \beta}+a B_{\alpha \mu} B_{\beta}^{\mu}+b G_{\alpha \beta} B^{\mu \nu} B_{\mu \nu} .
$$

Here $a$ and $b$ are constants to be determined and indices are raised with the GR metric $G_{\alpha \beta}, B^{\mu}{ }_{\beta}=G^{\mu \lambda} B_{\lambda \beta}$. With such an expansion the Lagrangian can be expanded as

$$
\mathbf{L}=\sqrt{-G}(R(G)+2 \Lambda)+\mathbf{L}_{B},
$$

where the first term is the Lagrangian corresponding to the GR background field with Ricci scalar $R$ and $\mathbf{L}_{B}$ is the contribution of the $B_{\alpha \beta}$ field, which acts then as a "matter" Lagrangian in the GR background field. In this formulation the $B$ gravitational stress tensor $T_{B}^{\mu \nu}$ is defined through the variation of the $B$ action according to [4] $\delta I_{B}=\int \delta \mathbf{L}_{B} d^{4} x=$ $8 \pi \int T_{B}^{\alpha \beta} \delta G_{\alpha \beta} d^{4} x$, that is,

$$
T_{B}^{\alpha \beta}=\frac{1}{8 \pi \sqrt{-G}} \frac{\delta \mathbf{L}_{B}}{\delta G_{\alpha \beta}} .
$$

All what we need is the $B$ field Lagrangian to second order and that is why we stopped at that order in Eq. (8). To make a close contact with I we rewrite Eq. (1) as a sum of a symmetric sector plus an antisymmetric one,

$\mathbf{L}=\mathbf{g}^{(\alpha \beta)}\left(U_{\alpha \beta}+\frac{1}{2} \Lambda g_{(\alpha \beta)}\right)+\mathbf{g}^{[\alpha \beta]}\left(\Gamma_{[\alpha, \beta]}+\frac{1}{2} \Lambda g_{[\alpha \beta]}\right)$

and consider the contribution of each term to $\mathbf{L}_{B}$. Only the contribution of the second term was considered in I, leading to a positive energy. Here we shall complete the calculation by investigating what the contribution of the first term is. Calling these contributions $\mathbf{L}_{1 B}$ and $\mathbf{L}_{2 B}$ we write $\mathbf{L}_{B}=\mathbf{L}_{1 B}+\mathbf{L}_{2 B}$ in Eq. (9),

$$
\mathbf{L}=\sqrt{-G}(R+2 \Lambda)+\mathbf{L}_{1 B}+\mathbf{L}_{2 B},
$$

where according to Eq. (11)

$\mathbf{L}_{1 B}=\mathbf{g}^{(\alpha \beta)}\left(U_{\alpha \beta}+\frac{1}{2} \Lambda g_{(\alpha \beta)}\right)-\sqrt{-G}\left(G^{\alpha \beta} R_{\alpha \beta}+2 \Lambda\right)$ and

$$
\mathbf{L}_{2 B}=\mathbf{g}^{[\alpha \beta]}\left(\Gamma_{[\alpha, \beta]}+\frac{1}{2} \Lambda g_{[\alpha \beta]}\right)
$$

Only this part of the Lagrangian was considered in I. To second order we see that this term is $\mathbf{L}_{2 B}^{(2)}=\sqrt{-G} B^{\alpha \beta}\left(\Gamma_{[\alpha, \beta]}^{(1)}+\frac{1}{2} \Lambda B_{\alpha \beta}\right)=-\Lambda \sqrt{-G}$ $G^{\alpha \gamma} G^{\beta \delta} B_{\gamma \delta} B_{\alpha \beta} / 2$, where use has been made of Eq. (5) in the last step. From Eq. (10) this gives to the $B$ stress tensor the contribution written in Eq. (I-5.19)

$$
T_{B}^{\alpha \beta}=-\frac{1}{8 \pi} \Lambda\left(\frac{1}{4} G^{\alpha \beta} B^{\mu \nu} B_{\mu \nu}-B^{\alpha \mu} B^{\beta}{ }_{\mu}\right),
$$

which is typical of a Maxwell field stress tensor if $\Lambda<0$. This then leads to a positive energy contribution as ascertained in I. By the way, this result follows immediately from the relations $\delta \sqrt{-G}=\sqrt{-G} G^{\alpha \beta} \delta G_{\alpha \beta} / 2$ and $\delta G^{\mu \rho}=$ $-G^{\mu(\alpha} G^{\beta) \rho} \delta G_{\alpha \beta}$ when varying $\mathbf{L}_{2 B}^{(2)}$.

Our problem now is to see what the contribution $\mathbf{L}_{1 B}$ of the $B$ field is. As it will be shown $\mathbf{L}_{1 B}$ will give no contribution to the $B$ stress tensor. We proceed with the expansion of the first term on the right of Eq. (13) on account of Eq. (8). First we have, with $B^{\mu \nu} B_{\mu \nu}=B^{2}$,

$$
\sqrt{-g}=\sqrt{-G}\left\{1+\frac{1}{4}(1-2 a+8 b) B^{2}\right\}
$$

Next, the inverse to Eq. (8) is $g^{\alpha \beta}=G^{\alpha \beta}+B^{\alpha \beta}+$ $(1-a) B^{\alpha \mu} B_{\mu}{ }^{\beta}-b G^{\alpha \beta} B^{2}$. Its symmetric part is $g^{(\alpha \beta)}=$ $G^{\alpha \beta}+(1-a) B^{\alpha \mu} B_{\mu}{ }^{\beta}-b G^{\alpha \beta} B^{2}$, whose inverse is $s_{\alpha \beta}=$ $G_{\alpha \beta}-(1-a) B^{\alpha \mu} B_{\mu} \beta+b G_{\alpha \beta} B^{2}$. Thus, from Eq. (3) we see that $\Gamma_{(\alpha \beta)}^{\sigma}=\Sigma_{\alpha \beta}^{\sigma}+\bar{\Gamma}_{(\alpha \beta)}^{\sigma}$ where the first term is the Christoffel connection of the $G$ field and the second term is the contribution of the $B$ field, which is at least of order $O\left(B^{2}\right)$ because $s_{\alpha \beta}=G_{\alpha \beta}+O\left(B^{2}\right)$. With this we obtain for $U_{\alpha \beta}$ the relation $U_{\alpha \beta}=R_{\alpha \beta}+\bar{U}_{\alpha \beta}$ where

$$
\bar{U}_{\alpha \beta}=\bar{\Gamma}_{(\alpha \beta) \mid \sigma}^{\sigma}-\bar{\Gamma}_{(\alpha \sigma) \mid \beta}^{\sigma}+\bar{\Gamma}_{(\alpha \beta)}^{\sigma} \bar{\Gamma}_{(\sigma \rho)}^{\rho}-\bar{\Gamma}_{(\alpha \rho)}^{\sigma} \bar{\Gamma}_{(\sigma \beta)}^{\rho}
$$

is the contribution of the $B$ field. Here a vertical bar indicates the Riemannian covariant derivative with respect to the background Christoffel connection. Knowing all this it is easy to write down the form of $\mathbf{L}_{1 B}$ in terms of $a$ and $b$. As each $\bar{\Gamma}$ is of order $O\left(B^{2}\right)$ we see that the term $\sqrt{-G} G^{\alpha \beta} \bar{U}_{\alpha \beta}$ coming from the expansion of the first term on the right-hand side of Eq. (13) is of fourth order in $B$, up to a total derivative coming from the two first terms of $\bar{U}_{\alpha \beta}$ which does not contribute to the Lagrangian. To the desired second order the final result is

$$
\mathbf{L}_{1 B}^{(2)}=\sqrt{-G}\left(\left\{\frac{1}{4}(1-2 a+4 b) B^{2} G^{\alpha \beta}+(1-a) B^{\alpha \mu} B_{\mu}{ }^{\beta}\right\} R_{\alpha \beta}+\Lambda(4 b-a) B^{2}\right) .
$$


The last task before calculating the corresponding contribution to the stress tensor is then to determine the values of the two parameters.

\section{Determination of the parameters}

To achieve the determination of the two parameters $a$ and $b$ we make use of the solution of the field equations for a point mass source found in [2] and compare with Eq. (8) when properly expanded. The solution for $g_{\alpha \beta}$ corresponding to the static spherically symmetric arc element, given in standard form by

$$
d s^{2}=\gamma d t^{2}-\alpha d r^{2}-r^{2}\left(d \theta^{2}+\sin ^{2} \theta d \phi^{2}\right),
$$

is

$$
\gamma=\left(1+\frac{F^{2}}{r^{4}}\right)^{1 / 2}-\frac{2 M}{r}\left(1+\frac{F^{2}}{r^{4}}\right)^{1 / 4}
$$

and

$$
\frac{1}{\alpha}=1+\frac{F^{2}}{r^{4}}-\frac{2 M}{r}\left(1+\frac{F^{2}}{r^{4}}\right)^{3 / 4}
$$

where $M$ is the mass of the particle. The last nonzero metric components are

$$
g_{[r 0]}=-g_{[0 r]}=\frac{F}{r^{2}} \frac{1}{\left(1+\frac{F^{2}}{r^{2}}\right)^{3 / 4}} .
$$

We shall now construct harmonic coordinates $X^{\alpha}$ out of the standard ones $x^{\alpha}=(t, r, \theta, \phi)$ following the same steps we encounter in GR [5]. These harmonic coordinates are the ones appropriate to radiation problems as we are now facing. The harmonic condition is here defined by the relation $\left(g^{(\alpha \beta)}(X) \Gamma_{(\alpha \beta)}^{\gamma}(X)\right)=0$ similar to GR. By a change to the coordinate $x^{\alpha}$ this relation becomes, as shown in Appendix A,

$$
g^{(\alpha \beta)} \frac{\partial^{2} X^{\lambda}}{\partial x^{\alpha} \partial x^{\beta}}-g^{(\alpha \beta)} \Gamma_{(\alpha \beta)}^{\gamma} X^{\lambda}, \gamma=0
$$

Notice that as Eq. (6) gives the relation $\mathbf{g}^{(\alpha \beta)}{ }_{\beta}+\mathbf{g}^{(\alpha \beta)} \Gamma_{(\alpha \beta)}^{\gamma}=0$ similar to GR, the above expression can also be written as $\left(\mathrm{g}^{(\alpha \beta)} X^{\lambda}, \alpha\right)_{, \beta}=0$. We set

$$
X^{1}=R(r) \sin \theta \cos \phi ; X^{2}=R(r) \sin \phi ; X^{3}=R(r) \cos \theta
$$

and look for the solution for $R(r)$ from Eq. (23). Having this we can determine the arc element in terms of the new space variables. Using $d r=d R / R, R^{2} d \Omega=d X_{i} d X_{i}-(d R)^{2}$, and $R d R=X_{i} d X_{i}$ we obtain

$$
d s^{2}=\gamma d t^{2}-\left[\frac{r^{2}}{R^{2}} \delta_{i j}+\left(\frac{\alpha}{R^{\prime 2}}-\frac{r^{2}}{R^{2}}\right) \frac{X_{i} X_{j}}{R^{2}}\right] d X^{i} d X^{j}
$$

Having $R(r)$ we will then be able to determine the parameters $a$ and $b$ by comparing the resultant metric components with Eq. (8). To obtain $R(r)$ we concentrate on Eq. (23) for the space component $X^{i}$ and make use of Eqs. (II-13) and (II-20) for the needed values of $g^{(\alpha \beta)}$ and $\Gamma_{(\alpha \beta)}^{\gamma}$. In this way we obtain a second order differential equation for $R(r)$ which we solve to first order in $F^{2} / r^{4}$ only. We neglect the curvature terms that depends on $M$ because the $F$ depen- dence is all we need for the determination of the parameters $a$ and $b$. We show the details of the calculation in Appendix $\mathrm{B}$ and quote here only the final result,

$$
R=r\left(1+\frac{F^{2}}{4 r^{4}}\right) .
$$

Plugging this in Eq. (25) together with the values of $\alpha$ and $\gamma$ in Eqs. (20) and (21) without the $M$ terms we find

$$
d s^{2}=\left(1+\frac{F^{2}}{2 r^{4}}\right) d t^{2}-\left[\left(1-\frac{F^{2}}{2 r^{4}}\right) \delta_{i j}+\frac{F^{2}}{r^{4}} \frac{X_{i} X_{j}}{R^{2}}\right] d X^{i} d X^{j}
$$

Now, to lowest order, that is keeping only $B_{0 i}$ terms and no curvature contributions, Eq. (8) gives

$$
g_{00}=1+(a-2 b) B_{0 i} B_{0 i}
$$

and

$$
g_{i j}=-\left(1-2 b B_{0 k} B_{0 k}\right) \delta_{i j}-a B_{0 i} B_{0 j} .
$$

Comparison with the metric components present in Eq. (27) shows that

$$
a=1
$$


and

$$
b=\frac{1}{4},
$$

and $B_{0 i} B_{0 i}=F^{2} / r^{4}$ this last relation being in agreement with the first order part of Eq. (22) $g_{[r 0]}^{(1)}=F / r^{2}=$ $\left(B_{0 i} B_{0 i}\right)^{1 / 2}$, the modulus of $B_{0 i}(r)$. With the results in Eqs. (30) and Eq. (31) we see the right hand side of Eq. (18) vanishes, $\mathbf{L}_{1 B}^{(2)}=0$. Therefore $\mathbf{L}_{1 B}$ is at least of fourth order in the $B$ field, and as a consequence of this the corresponding stress tensor vanishes. Therefore, Eq.(15) gives the full expression of the $B$ stress tensor. This then establishes in a complete way the positive definite character of the theory developed in [1].

\section{Appendix A: Harmonic relation}

The transformation formula for $g^{(\alpha \beta)}(x)$ when we go to a new coordinate frame $\bar{x}$ is

$$
g^{(\alpha \beta)}=\frac{\partial x^{\alpha}}{\partial \bar{x}^{\mu}} \frac{\partial x^{\beta}}{\partial \bar{x}^{\nu}} \bar{g}^{(\alpha \beta)}
$$

and for the symmetric part of the connection [6]

$$
\Gamma_{(\alpha \beta)}^{\gamma}=\frac{\partial x^{\alpha}}{\partial \bar{x}^{\eta}}\left(\frac{\partial \bar{x}^{\rho}}{\partial x^{\alpha}} \frac{\partial \bar{x}^{\sigma}}{\partial x^{\beta}} \bar{\Gamma}_{(\rho \sigma)}^{\eta}+\frac{\partial^{2} \bar{x}^{\eta}}{\partial x^{\alpha} \partial x^{\beta}}\right) .
$$

Contracting these two equations, and multiplying by $\partial \bar{x}^{\lambda} / \partial x^{\gamma}$ we obtain

$$
g^{(\alpha \beta)} \Gamma_{(\alpha \beta)}^{\gamma} \frac{\partial \bar{x}^{\lambda}}{\partial x^{\gamma}}=\bar{g}^{(\rho \sigma)} \bar{\Gamma}_{(\rho \sigma)}^{\lambda}+g^{(\alpha \beta)} \frac{\partial^{2} \bar{x}^{\lambda}}{\partial x^{\alpha} \partial x^{\beta}} .
$$

With the harmonic condition $\bar{g}^{(\rho \sigma)} \bar{\Gamma}_{(\rho \sigma)}^{\lambda}=0$ we obtain Eq. (23), with $\bar{x}^{\lambda}=X^{\lambda}$.

\section{Appendix B: Solution for $\mathbf{R}$}

We use Eqs. (II-13) and (II-20) for the needed values of $g^{(\alpha \beta)}$ and $\Gamma_{(\alpha \beta)}^{\gamma}$ and note in special the relations $g^{00} \gamma / \alpha=$ $\gamma /\left(\alpha \gamma-\omega^{2}\right)=-g^{11}$ and $\left(r \alpha g^{11}\right)^{-1}=1-F^{2} /\left(F^{2}+r^{4}\right)$. In this way we obtain after some algebra

$$
R "+R\left[\frac{\gamma^{\prime}}{2 \gamma}-\frac{\alpha^{\prime}}{2 \alpha}+\frac{2}{r}\left(1-\frac{F^{2}}{F^{2}+r^{4}}\right)\right]-\frac{2 \alpha R}{r^{2}}\left(1-\frac{F^{2}}{F^{2}+r^{4}}\right)=0
$$

which we solve for $R$ to first order in $F^{2} / r^{4}$ only. We neglect in Eqs. (20) and (21) the curvature terms that depends on $M$ because the $F$ dependence is all we need for the determination of the parameters $a$ and $b$. Putting $R=r+x F^{2}$ we are led to the differential equation

$$
x "+\frac{2 x^{\prime}}{r}-\frac{2 x}{r^{2}}-\frac{1}{r^{5}}=0,
$$

with solution $x=1 / 4 r^{3}$. Then, $R=r\left(1+F^{2} / 4 r^{4}\right)$ as written in Eq. (26)

\section{References}

[1] S. Ragusa, Phys. Rev. D 56, 864 (1997). Eq. (n) of this paper will be referred to as Eq. (I-n).
[2] S. Ragusa, Gen. Relat. Grav. 31, 275 (1999). Eq. (n) of this paper will be referred to as Eq. (II-n).

[3] T. Damour, S. Deser and J. MaCarthy, Phys. Rev. D 45, R3289 (1992); 47, 1541 (1993).

[4] L. D. Landau and E. M. Lifshitz, The Classical Theory of Fields (Pergamon, Oxford, England, 1975).

[5] S. Weinberg, Gravitation and Cosmology (Wiley, New York, 1972).

[6] A. Papapetrou, Lectureson General Relativity (Dordrecht, Holland,1974). The transformation property for $\Gamma_{\alpha \beta}^{\gamma}$ given here is general, valid for the nonsymmetric case. 